\title{
Schmerzen per Ultraschall schonend aufspüren - und gezielt therapieren
}

Über 12 Millionen Menschen leiden hierzulande unter chronischen Schmerzen. Die Ursache dafür zu finden, ist oft schwierig - häufig dauert es mehrere Jahre, bis Patienten eine wirksame Behandlung erhalten. Doch moderne Ultraschalltechniken geben Hoffnung: Mit den Verfahren können einerseits sogar kleinste Nervenäste und andererseits durch dynamische und an die jeweilige Anatomie angepasste Untersuchung der Ort der Schmerzauslösung erkannt - und damit Schmerzursachen exakt diagnostiziert und zugeordnet werden. Wie funktioniert das? Und inwiefern kommen moderne Ultraschalltechniken in der Schmerztherapie zum Einsatz? Antworten auf diese und weitere Fragen gaben Experten der Deutschen Gesellschaft für Ultraschall in der Medizin e. V. (DEGUM) am 11. Dezember 2019 auf einer Pressekonferenz in Berlin.

Zu Beginn der Veranstaltung berichtete PD Dr. med. Christian Tesch, Leiter der DEGUM-Sektion Chirurgie, wie moderne Ultraschalltechniken in der Therapie von chronischen Schmerzen eingesetzt werden. „Wenn Patienten beispielsweise Beschwerden in der Umgebung der Wirbelsäule haben, können Schmerzmittel unter Ultraschallkontrolle gezielt gegeben werden - das war bisher nur durch Computertomografie oder per Bildwandlerkontrolle möglich. Da bei diesen Verfahren jedoch Röntgenstrahlung zum Einsatz kommt, sind sie im Gegensatz zum Ultraschall gesundheitsschädigend.“

\section{Schmerzen in kleinsten \\ Nervenästen diagnostizieren - Ultraschall macht's möglich}

Anschließend erläuterte Dr. med. Carla Alessandra Ávila González, stellvertretende Leiterin der DEGUM-Sektion Anästhesiologie, wie Ultraschallverfahren bei der Diagnose von chronischen Schmerzen zum Einsatz kommen. „Wenn die Schmerzursache nicht durch klinische Anamnese und Untersuchung gesichert werden kann und es wahrscheinlich ist, dass eine lokale Schädigung - beispielsweise von Nerven, Muskeln oder Gefäßen - die Ursache ist, ist eine Ultraschalluntersuchung empfehlenswert“, sagte Ávila González. Dabei wird jede Gewebeschicht - also Fettgewebe, Faszien, Muskulatur und Knochen - mit unterschiedlich dosiertem Druck sowohl händisch als auch per Ultraschallsonde gezielt untersucht. „So wird die Schmerzempfindlichkeit unterschiedlicher anatomischer Strukturen, wie beispielsweise der Nerven, Muskeln oder Sehnen, exakt beurteilt. Dank der neuesten Generation von Ultraschallgeräten und Sonden mit Untersuchungsfrequenzen oberhalb von 10 bis zu 30 Megahertz können mittlerweile sogar

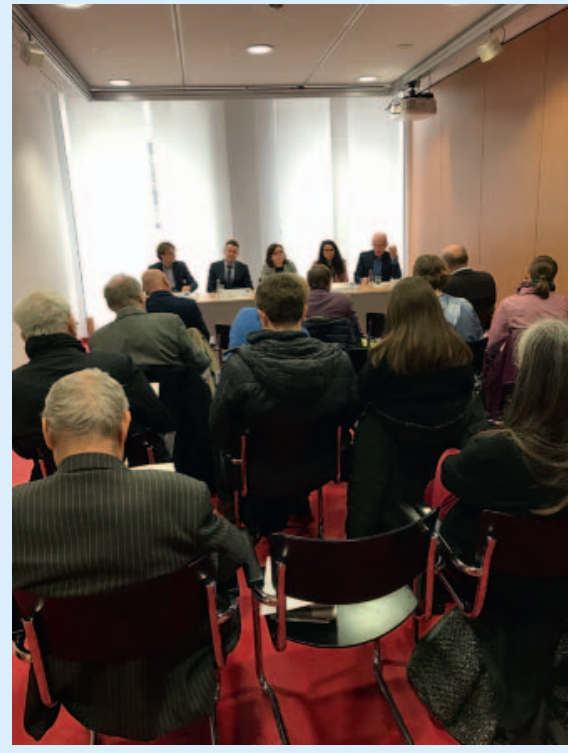

kleinste Nervenstrukturen von unter 0,1 Millimetern dargestellt werden“, so die DEGUM-Expertin. Sogar das Binnenmuster der Nerven könne so auf mögliche krankhafte Veränderungen - etwa auf Nerventumoren - hin untersucht werden.

\section{Wie Kinderrheuma mittels Ultraschalls sicher diagnostiziert werden kann}

Auch beim Verdacht auf Kinderrheuma kann die Sonografie angewendet werden. Wie das funktioniert, erklärte DEGUMExperte PD Dr. med. Daniel Windschall. 
„Durch die Entwicklung der Ultraschalltechnik im Nahbereich können insbesondere Gelenke von Kindern hervorragend geschallt und sehr einfach und schnell untersucht werden." Studien hätten gezeigt, dass die Sonografie zum Nachweis einer Gelenkentzündung bei Kindern sogar genauere und bessere Ergebnisse liefere als die äußerliche Gelenkuntersuchung. Somit sollten Ärzte den Ultraschall zusätzlich zur äußerlichen klinischen Untersuchung einsetzen.

\section{Das Karpaltunnelsyndrom genau aufspüren - warum der Ultraschall besonders Erfolg versprechend ist}

Zum Abschluss der Pressekonferenz referierte DEGUM-Experte Dr. med. Henrich Kele über den Einsatz der Sonografie beim Karpaltunnelsyndrom. „Moderne, hochauflösende Ultraschallgeräte können den betroffenen Nerv sehr detailliert abbilden und liefern deshalb beim Karpaltunnelsyndrom - ergänzend zur regulär durchgeführten elektrophysiologischen Untersuchung exakte Informationen über die Lage der Engstelle und über die Ursache“, so Kele. Diese Informationen seien entscheidend für eine möglichst effektive Behandlung und die Entscheidung für oder gegen eine Operation. 